\title{
MIXTURE REPRESENTATIONS FOR THE JOINT DISTRIBUTION OF LIFETIMES OF TWO COHERENT SYSTEMS WITH SHARED COMPONENTS
}

\author{
JORGE NAVARRO, ${ }^{*}$ Universidad de Murcia \\ FRANCISCO J. SAMANIEGO, ${ }^{* *}$ University of California, Davis \\ N. BALAKRISHNAN, ${ }^{* * *}$ McMaster University and King Abdulaziz University
}

\begin{abstract}
The signature of a system is defined as the vector whose $i$ th element is the probability that the system fails concurrently with the $i$ th component failure. The signature vector is known to be a distribution-free measure and a representation of the system's survival function has been developed in terms of the system's signature. The present work is devoted to the study of the joint distribution of lifetimes of pairs of systems with shared components. Here, a new distribution-free measure, the 'joint bivariate signature', of a pair of systems with shared components is defined, and a new representation theorem for the joint survival function of the system lifetimes is established. The theorem is shown to facilitate the study of the dependence between systems and the comparative performance of two pairs of such systems.
\end{abstract}

Keywords: Coherent system; $k$-out-of- $n$ system; order statistics; signature; mixture; stochastic order

2010 Mathematics Subject Classification: Primary 60E15

Secondary $60 \mathrm{~K} 10$

\section{Introduction}

The signature of a coherent system with $n$ components having independent and identically distributed (i.i.d.) lifetimes $X_{1}, \ldots, X_{n} \sim F$, where $F$ is the common continuous distribution function, was introduced by Samaniego [30] as the $n$-dimensional vector $\boldsymbol{p}$ whose $i$ th element is given by

$$
p_{i}=\mathbb{P}\left(T=X_{i: n}\right), \quad i=1, \ldots, n,
$$

where $X_{1: n}, \ldots, X_{n: n}$ are the order statistics corresponding to the $n$ component lifetimes and $T$ is the system lifetime. A $k$-out-of- $n$ system (defined as the system which fails upon the $k$ th component failure) has the unit vector $(0, \ldots, 1, \ldots, 0)$ as its signature vector, where the value ' 1 ' occurs as the $k$ th element.

If the component lifetimes have a common continuous distribution $F$ then distribution $F_{T}$ of the coherent system with signature vector $\boldsymbol{p}$ and lifetime $T$ can be represented as a mixture of

\footnotetext{
Received 5 October 2012; revision received 5 February 2013.

* Postal address: Facultad de Matemáticas, Universidad de Murcia, 30100 Murcia, Spain.

Email address: jorgenav@um.es

** Postal address: Department of Statistics, University of California, Davis, 1 Shields Avenue, Davis, CA 95616, USA.

Email address: fjsamaniego@ucdavis.edu

*** Postal address: Department of Mathematics and Statistics, McMaster University, Hamilton, Ontario L8S 4K1,

Canada. Email address: bala@mcmaster.ca
} 
the distributions $F_{i: n}$ of the ordered component lifetimes $X_{i: n}, i=1, \ldots, n$, with the weights given in the signature vector, that is,

$$
F_{T}(t)=\sum_{i=1}^{n} p_{i} F_{i: n}(t), \quad t \geq 0 .
$$

The distribution-free signature vector can also be computed as

$$
p_{i}=\frac{\left|A_{i}\right|}{n !}, \quad i=1, \ldots, n,
$$

where $\left|A_{i}\right|$ is the size of the set $A_{i}=\left\{\sigma \in \mathcal{P}_{n}: T=X_{i: n}\right.$ when $\left.X_{\sigma(1)}<\cdots<X_{\sigma(n)}\right\}$ and $\mathcal{P}_{n}$ is the set of permutations of the set $\{1, \ldots, n\}$.

Kochar et al. [17] used representation (1.1) to compare systems with different structures by comparing their signature vectors. They established several preservation theorems showing that various types of stochastic relationship between system signatures are inherited by the system lifetimes. For an introduction to system signatures and their applications, see the monograph by Samaniego [31]. Recent developments in this area include [7], [14], [20], [21], [24], [25], [27], [29], and [32].

A representation similar to (1.1) holds for the system's reliability as well as for systems with exchangeable components (see Theorem 3.2 of [29]). For any $n>k$, the distribution of a given coherent system in $k$ i.i.d. components $(\sim F)$ can be represented as (1.1) for some nonnegative coefficients $p_{1}, \ldots, p_{n}$ (see Theorem 3.4 of [29]). The vector with those coefficients is said to be its signature of order $n$. Samaniego [31, p. 32] presented an expression for the signature of order $k+1$ from the signature of order $k$. Navarro et al. [29] provided an explicit formula for the signature of order $n$ for arbitrary $n>k$. The signatures of order $n$ are used in [29] to compare systems of different sizes $n$ and $k$, where $n>k$.

Systems with shared components arise in a variety of applications. Computer systems consisting of slave computers sharing a common server is perhaps the quintessential example. The present paper is intended as a contribution to the literature on such systems. The results obtained here apply both to pairs of systems which share a common module (i.e. a specific configuration of the common components) and to pairs of systems having different structures but being dependent on one or more components which the two systems have in common. An example of the latter situation is a pair of systems (say, buildings) supported by multiple power generators, some of which serve both systems and some of which solely support one or the other system but not both. The configuration of the generators serving each system may be structured according to wholly different designs.

The present results will also be useful to study dependence between a system lifetime $T=$ $\psi\left(X_{1}, \ldots, X_{n}\right)$, where $\psi$ is the system's structure function (see, for example, [5, p. 12]), and the component lifetimes $X_{1}, \ldots, X_{n}$. For example, we might be interested in studying the dependence between $T$ and the earliest component failure time $X_{1: n}=\min \left(X_{1}, \ldots, X_{n}\right)$ or a specific component failure time $X_{i}$ for $i \in\{1, \ldots, n\}$.

Under the assumption that all components in two systems with shared components have i.i.d. lifetimes with common continuous distribution $F$, Navarro et al. [26] established an explicit representation of the joint distribution of the pair $\left(T_{1}, T_{2}\right)$ of system lifetimes. The representation in [26] displays the joint distribution $G\left(t_{1}, t_{2}\right)=\mathbb{P}\left(T_{1} \leq t_{1}, T_{2} \leq t_{2}\right)$ of system lifetimes separately, for $t_{1} \leq t_{2}$ or for $t_{1}>t_{2}$, as a function of distribution-free matrices of coefficients $S$ or $S^{*}$ and the marginal distributions of the order statistics of component lifetimes. 
Worthy of special mention is the fact that the representations in [26] do not involve the joint behavior of these order statistics. As we will see, this is a key difference between this representation and the main result in the present work. While the matrix pair $\left(S, S^{*}\right)$ in [26] depends solely on the two system designs, and the elements of each matrix must sum to 1 , the authors pointed out that they need not be nonnegative and, thus, do not have, in general, a probabilistic interpretation. The representation in [26] has nonetheless been shown to be useful in establishing sufficient conditions for the (bivariate) lower- or upper-orthant ordering between the joint lifetimes of two pairs of systems with shared components. A challenge left for future research is to identify sufficient conditions for the usual bivariate stochastic ordering between competing pairs of systems, each having some extent of component sharing. A solution to this challenge is provided in the present work, with the new representation theorem in Section 2 serving as the key tool for this specific application.

In Section 2 we establish a new representation theorem for the joint distribution of the lifetimes $\left(T_{1}, T_{2}\right)$ of two coherent systems with shared components. We will assume, as in [26], that the components of two systems are overlapping subsets of a set of $n$ components with i.i.d. lifetimes having a common continuous distribution $F$; we also assume, without loss of generality, that the union of the sets of components utilized by each of the two systems is equal to the set of $n$ components alluded to above. No restriction is made on the design or structure of the two systems. Theorem 2.1 utilizes the joint distribution of pairs $\left(X_{i: n}, X_{j: n}\right)$ of ordered component lifetimes and yields a markedly different representation of the joint distribution of $\left(T_{1}, T_{2}\right)$ than the one given in [26]. Specifically, the representation involves a stochastic rather than generalized mixture of bivariate distributions of pairs of ordered component lifetimes and features a new distribution-free measure, the bivariate signature matrix (BSM), with the useful characteristic of having nonnegative elements summing to 1 and, thus, having an immediate probabilistic interpretation. Most importantly, the matrix orderings of this new measure are shown to provide the sought-after sufficient conditions for the bivariate stochastic ordering of competing pairs of systems with shared components under the framework outlined above. Also treated is the comparison of two pairs of systems with shared components relative to stochastic precedence. A further notable feature of the new result is that it provides a linear representation of $G\left(t_{1}, t_{2}\right)$ for arbitrary $t_{1}$ and $t_{2}$, while the result in [26] requires separate expressions for $t_{1} \leq t_{2}$ and $t_{1}>t_{2}$.

Multidimensional signatures have been considered previously in [10], [11], [12], and [13] under the assumption that both systems have the same set of components, where the lifetimes $T_{1}$ and $T_{2}$ correspond to the transition times from the system UP state to DOWN1 and DOWN2 states, respectively. Gertsbakh and Shpungin [12] discussed the notion of bivariate signatures. While they did not treat possible representation theorems, they did provide an algorithm for estimating the BSM from Monte Carlo simulations. To our knowledge, the representation theorems presented here, and the applications which follow, are new to the literature. Our results were first presented at the ISI World Statistics Conference in Dublin, Ireland in 2011.

The paper is organized as follows. In Section 2, the definition of the BSM, the statement and proof of Theorem 2.1, which yield a new representation theorem for the joint lifetimes $\left(T_{1}, T_{2}\right)$ of two coherent systems with shared components, and the development of new results on the dependence between $T_{1}$ and $T_{2}$ for such systems are presented. In Section 3 , the BSM of each of two pairs of systems with shared components based on a pool of $n$ components with i.i.d. lifetimes is shown to be a tool that is ideally suited for obtaining various ordering results between the two pairs of systems. Specifically, the representation in Theorem 2.1 is utilized in establishing a heretofore elusive result, namely, the identification of sufficient conditions 
for bivariate stochastic ordering of pairs of such systems. Furthermore, the bivariate signature matrices of two such pairs of systems are shown to be useful in comparing these systems in terms of stochastic precedence. Four concrete examples which illustrate our theoretical results are provided in the final section.

\section{Main results}

Let us assume that $T_{1}$ and $T_{2}$ are the lifetimes of two coherent systems based on components with i.i.d lifetimes $X_{1}, \ldots, X_{n}$ having a common continuous distribution function $F$. We are particularly interested in the case in which the two systems share one or more components, and we will allow for each of the systems to be of order less than $n$. For the sake of clarity, one can think of the two systems of interest as being based on $n_{1}$ and $n_{2}$ components and having $n_{1,2}$ components in common (i.e. having $n_{1,2}$ shared components). We will assume that $n_{1,2}>0$, as otherwise the lifetimes of the two systems are independent (under the i.i.d. assumption made above), and the joint distribution of their lifetimes is simply the product of marginal distributions of the form shown in (1.1). We may identify the integer $n$ above uniquely as $n=n_{1}+n_{2}-n_{1,2}$. We also consider the case in which these systems are based just on some of these component lifetimes and not on all of them. So these two systems might share all, some, or none of these components. It is well known that a coherent system fails at the failure of one of its components. Let us denote the (increasing) ordered component lifetimes as $X_{1: n}, \ldots, X_{n: n}$. Note that, using the i.i.d. assumption and the continuity of $F$, we have $\mathbb{P}\left(X_{1: n}<\cdots<X_{n: n}\right)=1$. Hence, we can define the random vector $\boldsymbol{I}=\left(I_{1}, I_{2}\right)$ by

$$
\boldsymbol{I}=(i, j) \quad \text { whenever } \quad T_{1}=X_{i: n} \quad \text { and } \quad T_{2}=X_{j: n} .
$$

The bivariate probability mass function of $\boldsymbol{I}$ is denoted by $p_{i, j}=\mathbb{P}(\boldsymbol{I}=(i, j))$ for $i, j=$ $1, \ldots, n$. Note that

$$
p_{i, j}=\frac{\left|A_{i, j}\right|}{n !}
$$

where $\left|A_{i, j}\right|$ is the size of the set

$$
A_{i, j}=\left\{\sigma \in \mathcal{P}_{n}: T_{1}=X_{i: n} \text { and } T_{2}=X_{j: n} \text { whenever } X_{\sigma(1)}<\cdots<X_{\sigma(n)}\right\}
$$

and $\mathcal{P}_{n}$ is the set of permutations of the set $\{1, \ldots, n\}$.

Definition 2.1. The matrix $\boldsymbol{P}=\left(p_{i, j}\right)$ is called the bivariate signature matrix $(B S M)$ associated with $\left(T_{1}, T_{2}\right)$.

The following properties of the BSM are immediately evident.

(P1) The bivariate signature $\boldsymbol{P}=\left(p_{i, j}\right)$ does not depend on $F$. Actually, it can be computed using (2.2) in the same manner as the univariate signature can be computed from (1.2) by considering the $n$ ! permutations of the $n$ component failure times. This procedure is illustrated in Example 4.1 below.

(P2) Of course, we have $p_{i, j} \geq 0$ and $\sum_{i=1}^{n} \sum_{j=1}^{n} p_{i, j}=1$. Moreover, the univariate signature of order $n,\left(p_{1}, \ldots, p_{n}\right)$, of the coherent system with lifetime $T_{1}$ can be computed from the BSM as $p_{i}=\sum_{j=1}^{n} p_{i, j}$ (i.e. as the marginal distribution of $I_{1}$ ). Analogously, the signature of order $n,\left(p_{1}^{*}, \ldots, p_{n}^{*}\right)$, of $T_{2}$ can be computed as $p_{j}^{*}=\sum_{i=1}^{n} p_{i, j}$. In particular, if the first system has $n$ components then the 'usual' one-dimensional signature of the first system can be obtained by taking the corresponding marginal of the $\left\{p_{i j}\right\}$ matrix (see the examples in Section 4). 
(P3) If $T_{2}=X_{k: n}$ (i.e. $T_{2}$ is the lifetime of a $k$-out-of- $n$ system) then $p_{i, k}=p_{i}$ and $p_{i, j}=0$ for $i=1, \ldots, n$ and $j \neq k$, where $\left(p_{1}, \ldots, p_{n}\right)$ is the signature of order $n$ of $T_{1}$. In this case, $I_{1}$ and $I_{2}$ are independent.

The main property of the BSM is given in the theorem below where it is proven that the joint distribution of two coherent systems sharing some (or all) components can be written as a stochastic mixture of the bivariate joint distributions of the ordered component lifetimes. This representation is similar to the representation in (1.1) obtained for a single coherent system in [30].

Theorem 2.1. Let $T_{1}$ and $T_{2}$ be the lifetimes of two coherent systems respectively based on $n_{1}$ and $n_{2}$ i.i.d. components with a common continuous distribution $F$. Assume that they have $n_{1,2} \geq 0$ components in common. Then, the joint distribution function $G\left(t_{1}, t_{2}\right)=\mathbb{P}\left(T_{1} \leq t_{1}\right.$, $\left.T_{2} \leq t_{2}\right)$ of $\left(T_{1}, T_{2}\right)$ can be written as

$$
G\left(t_{1}, t_{2}\right)=\sum_{i=1}^{n} \sum_{j=1}^{n} p_{i, j} F_{i, j: n}\left(t_{1}, t_{2}\right)
$$

where $n=n_{1}+n_{2}-n_{1,2}, \boldsymbol{P}=\left(p_{i, j}\right)$ is the BSM of $\left(T_{1}, T_{2}\right)$, and $F_{i, j: n}\left(t_{1}, t_{2}\right)=\mathbb{P}\left(X_{i: n} \leq t_{1}\right.$, $\left.X_{j: n} \leq t_{2}\right)$ is the joint distribution of the order statistics obtained from the component lifetimes $X_{1}, \ldots, X_{n}$.

Proof. As $F$ is continuous, using the theorem of total probability, we have

$$
\begin{aligned}
G\left(t_{1}, t_{2}\right) & =\mathbb{P}\left(T_{1} \leq t_{1}, T_{2} \leq t_{2}\right) \\
& =\sum_{i=1}^{n} \sum_{j=1}^{n} \mathbb{P}(\boldsymbol{I}=(i, j)) \mathbb{P}\left(T_{1} \leq t_{1}, T_{2} \leq t_{2} \mid \boldsymbol{I}=(i, j)\right) \\
& =\sum_{i=1}^{n} \sum_{j=1}^{n} p_{i, j} \mathbb{P}\left(X_{i: n} \leq t_{1}, X_{j: n} \leq t_{2} \mid \boldsymbol{I}=(i, j)\right) \\
& =\sum_{i=1}^{n} \sum_{j=1}^{n} p_{i, j} \mathbb{P}\left(X_{i: n} \leq t_{1}, X_{j: n} \leq t_{2}\right),
\end{aligned}
$$

where the last equality follows since, under the i.i.d. assumption, the events $\left\{X_{i: n} \leq t_{1}\right.$, $\left.X_{j: n} \leq t_{2}\right\}$ and $\{\boldsymbol{I}=(i, j)\}$ are independent. Hence, the proof is completed.

The joint distribution $G$ of $\left(T_{1}, T_{2}\right)$ has a singular part whenever $\mathbb{P}\left(T_{1}=T_{2}\right)>0$. However, if the components are i.i.d. $\sim F$ and $F$ is absolutely continuous, then $F_{i: n}\left(t_{1}\right) F_{j: n}\left(t_{2}\right)$ and $F_{i, j: n}\left(t_{1}, t_{2}\right)$ are both absolutely continuous bivariate distributions when $i \neq j$. Therefore, the joint distribution $G$ of $\left(T_{1}, T_{2}\right)$ cannot be represented as a mixture of these bivariate distributions. Actually, in [26], we needed two different linear combinations (one for $t_{1} \leq t_{2}$ and another for $\left.t_{1}>t_{2}\right)$ based on $F_{i: n}\left(t_{1}\right) F_{j: n}\left(t_{2}\right)$ to obtain $G$ and to include its singular part. In the preceding theorem, as

$$
F_{i, i: n}\left(t_{1}, t_{2}\right)=\mathbb{P}\left(X_{i: n} \leq t_{1}, X_{i: n} \leq t_{2}\right)=F_{i: n}\left(\min \left(t_{1}, t_{2}\right)\right)
$$

is a singular bivariate distribution (the joint distribution of $\left.\left(X_{i: n}, X_{i: n}\right)\right)$, we can represent $G$ by using the linear combination (2.3) for all $t_{1}, t_{2}$. In particular, $G$ is absolutely continuous if and only if $p_{i, i}=0$ for all $i=1, \ldots, n$. In this case, its density function $g$ 
can be written as $g\left(t_{1}, t_{2}\right)=\sum_{i=1}^{n} \sum_{j=1}^{n} p_{i, j} f_{i, j: n}\left(t_{1}, t_{2}\right)$, where $f_{i, j: n}$ is the joint density of $\left(X_{i: n}, X_{j: n}\right)$ for $i \neq j$. A similar representation can be stated for the joint reliability function of $\left(T_{1}, T_{2}\right)$ with the same coefficients as $\bar{G}\left(t_{1}, t_{2}\right)=\sum_{i=1}^{n} \sum_{j=1}^{n} p_{i, j} \bar{F}_{i, j: n}\left(t_{1}, t_{2}\right)$, where $\bar{F}_{i, j: n}\left(t_{1}, t_{2}\right)=\mathbb{P}\left(X_{i: n}>t_{1}, X_{j: n}>t_{2}\right)$.

The functions $F_{i: n}, F_{i, j: n}, \bar{F}_{i, j: n}$, and $f_{i, j: n}$ can all be computed from $F$ using the expressions known in the theory of order statistics (see, e.g. [8, pp. 9-12]). For example, if $1 \leq i<j \leq n$ then $F_{i, j: n}$ can be computed as

$$
F_{i, j: n}\left(t_{1}, t_{2}\right)=\sum_{r=j}^{n} \sum_{s=i}^{r} \frac{n !}{s !(r-s) !(n-r) !} F^{s}\left(t_{1}\right)\left(F\left(t_{2}\right)-F\left(t_{1}\right)\right)^{r-s}\left(1-F\left(t_{2}\right)\right)^{n-r}
$$

for $t_{1}<t_{2}$, and

$$
F_{i, j: n}\left(t_{1}, t_{2}\right)=F_{j: n}\left(t_{2}\right)=\sum_{r=j}^{n} \frac{n !}{r !(n-r) !} F^{r}\left(t_{2}\right)\left(1-F\left(t_{2}\right)\right)^{n-r}
$$

for $t_{1} \geq t_{2}$. Expanding these expressions in terms of powers of $F$ (or $\bar{F}=1-F$ ), and using the expressions in [8, p. 46], we obtain the expressions for $G$ obtained in [26].

The univariate signature can be used to compute the moments of a single coherent system lifetime from (1.1). Analogously, the bivariate signature can be used to compute product moments and covariance coefficients as follows.

Theorem 2.2. Under the assumptions of Theorem 2.1, if $T_{1}$ and $T_{2}$ have respective signatures $\left(p_{1}, \ldots, p_{n}\right)$ and $\left(p_{1}^{*}, \ldots, p_{n}^{*}\right)$ of order $n$ and $B S M \boldsymbol{P}=\left(p_{i, j}\right)$, then

$$
\mathbb{E}\left(T_{1} T_{2}\right)=\sum_{i=1}^{n} p_{i, i} \alpha_{i, i: n}+\sum_{i=1}^{n} \sum_{j=i+1}^{n}\left(p_{i, j}+p_{j, i}\right) \alpha_{i, j: n}
$$

and

$$
\operatorname{cov}\left(T_{1}, T_{2}\right)=\sum_{i=1}^{n} \sum_{j=1}^{n} p_{i, j} \sigma_{i, j: n}+\sum_{i=1}^{n} \sum_{j=1}^{n}\left(p_{i, j}-p_{i} p_{j}^{*}\right) \mu_{i: n} \mu_{j: n},
$$

where $\mu_{i: n}=\mathbb{E}\left(X_{i: n}\right), \alpha_{i, j: n}=\mathbb{E}\left(X_{i: n} X_{j: n}\right), \sigma_{i, j: n}=\operatorname{cov}\left(X_{i: n}, X_{j: n}\right)$, and $\sigma_{i, i: n}=\sigma_{i: n}^{2}=$ $\operatorname{var}\left(X_{i: n}\right)$ for $i, j=1, \ldots, n$.

The proof is straightforward. In particular, if one system is a $k$-out-of- $n$ system, that is, e.g. $T_{2}=X_{k: n}$, then, from (P3), we have $p_{i, k}=p_{i}$ for $i=1, \ldots, n$ and $p_{i, j}=0$ for $i, j=1, \ldots, n$ and $j \neq k, \mathbb{E}\left(T_{1} X_{k: n}\right)=\sum_{i=1}^{n} p_{i} \alpha_{i, k: n}$, and

$$
\operatorname{cov}\left(T_{1}, X_{k: n}\right)=\sum_{i=1}^{k-1} p_{i} \sigma_{i, k: n}+p_{j} \sigma_{k: n}^{2}+\sum_{i=k+1}^{n} p_{i} \sigma_{i, k: n}
$$

Particular expressions can be obtained when the component lifetimes have a common exponential distribution. In this case, it is well known (see [9]) that $\operatorname{cov}\left(X_{i: n}, X_{j: n}\right)=\operatorname{var}\left(X_{i: n}\right)$ for $j \geq i$. This property can be extended as follows. The proof is given in [28], which is available from the first author upon request.

Corollary 2.1. Under the assumptions of Theorem 2.1 , if $T_{1}$ has signature $\left(0, \ldots, 0, p_{k}\right.$, $\left.\ldots, p_{n}\right)$ of order $n$ and the i.i.d component lifetimes have a common exponential distribution, then

$$
\operatorname{cov}\left(T_{1}, X_{j: n}\right)=\operatorname{var}\left(X_{j: n}\right) \text { for } j=1, \ldots, k \text {. }
$$


We now turn to the study of the conditional distribution of a system when we know that the $j$ th component failure occurs at time $x>0$.

Theorem 2.3. Let $T$ be the lifetime of a coherent system with i.i.d. components having a common continuous distribution $F$ and with signature of order $n$ of the form $\left(0, \ldots, 0, p_{k}, \ldots, p_{n}\right)$. Then the distribution function of $\left(T-x \mid X_{j: n}=x\right)$ for $1 \leq j<k$ and $x>0$ can be written as

$$
G(y \mid x)=\sum_{i=k}^{n} p_{i} H_{i-j \mid n-j}(y \mid x),
$$

where $H_{i-j \mid n-j}(y \mid x)$ is the distribution function of the $(i-j)$ th order statistic from $n-j$ i.i.d. random variables with common distribution function $H(y \mid x)=(F(x+y)-F(x)) /(1-F(x))$ for $y \geq 0$.

Proof. From property (P3) and Theorem 2.1, the joint density function of $\left(X_{j: n}, T\right)$ is given by $g(x, y)=\sum_{i=k}^{n} p_{i} f_{j, i: n}(x, y)$, where $f_{j, i: n}(x, y)$ is the joint density of $\left(X_{j: n}, X_{i: n}\right)$. Hence, the density function of $\left(T-x \mid X_{j: n}=x\right)$ can be written as

$$
g(y \mid x)=\frac{g(x, x+y)}{f_{j: n}(x)}=\sum_{i=k}^{n} p_{i} f_{i \mid j: n}(y \mid x),
$$

where $f_{i \mid j: n}(y \mid x)=f_{j, i: n}(x, x+y) / f_{j: n}(x)$ is the density function of $\left(X_{i: n}-x \mid X_{j: n}=x\right)$. From Equation (3) of [4], a straightforward calculation gives

$$
\begin{aligned}
f_{i \mid j: n}(y \mid x) & \\
= & \frac{f_{j, i: n}(x, x+y)}{f_{j: n}(x)} \\
= & \frac{(n-j) !}{(n-i) !(i-j-1) !}\left(\frac{F(x+y)-F(x)}{1-F(x)}\right)^{i-j-1}\left(\frac{1-F(x+y)}{1-F(x)}\right)^{n-i} \frac{f(x+y)}{1-F(x)} \\
= & \frac{(n-j) !}{(n-j-(i-j)) !(i-j-1) !} H^{i-j-1}(y \mid x)(1-H(y \mid x))^{n-j-(i-j)} h(y \mid x),
\end{aligned}
$$

where $H(y \mid x)=(F(x+y)-F(x)) /(1-F(x))$ and $h(y \mid x)=f(x+y) /(1-F(x))$. Hence, $f_{i \mid j: n}(y \mid x)=h_{i-j: n-j}(y \mid x)$, where $h_{i-j: n-j}(y \mid x)$ is the density function of the $(i-j)$ th order statistic from $n-j$ i.i.d. random variables with common distribution function $H(y \mid x)$ for $y \geq 0$. Therefore, from the expression obtained for $g(y \mid x)$, the proof is complete.

Note that we have proved that $\left(T-x \mid X_{j: n}=x\right)$ is equal in law (distribution) to the lifetime of a coherent system in $n-j$ i.i.d. components with common distribution function $H(y \mid x)$ and signature of order $n-j$ given by $\left(0, \ldots, 0, p_{k}, \ldots, p_{n}\right)$. A similar expression holds for the reliability functions. Other representations under different conditions (such as $X_{j: n} \leq x$ or $X_{j: n}>x$ ) have been obtained in [2], [3], [6], [16], [18], [19], [22], [25], [32], [34], and [35].

The property in Theorem 2.3 can be extended using the Markovian property of order statistics, revealing that, for any $j<k,\left(T-t_{j} \mid X_{1: n}=t_{1}, \ldots, X_{j: n}=t_{j}\right)$ is equal in law to $\left(T-t_{j} \mid X_{j: n}=t_{j}\right)$. In particular, for systems with exponential components, we have the following memoryless property: if $T$ has signature $\left(0, \ldots, 0, p_{k}, \ldots, p_{n}\right)$ of order $n$ and i.i.d. exponential components, then the distribution function of $\left(T-x \mid X_{j: n}=x\right)$ does not depend on $x$ and can be written as

$$
\mathbb{P}\left(T-x \leq y \mid X_{j: n}=x\right)=\sum_{i=k}^{n} p_{i} F_{i-j: n-j}(y),
$$


where $F_{i-j: n-j}(y)$ is the distribution of the $(i-j)$ th order statistic from $n-j$ i.i.d. random variables with a common exponential distribution function $F$. Hence, we have $\mathbb{E}\left(T \mid X_{j: n}=\right.$ $x)=x+\mathbb{E}\left(T^{*}\right)$, where $\mathbb{E}\left(T^{*}\right)=\sum_{i=k}^{n} p_{i} \mu_{i-j: n-j}$ and $\mu_{i-j: n-j}=\mathbb{E}\left(X_{i-j: n-j}\right)$. Note that $\mu_{i-j: n-j}$ does not depend on $x$ and is given by $\mu_{i-j: n-j}=\sum_{r=1}^{i-j} \mu /(n-j-r+1)$. Therefore,

$$
\mathbb{E}\left(T \mid X_{j: n}=x\right)=x+\sum_{i=k}^{n} p_{i} \mu_{i-j: n-j}=x+\sum_{i=k}^{n} p_{i} \sum_{r=1}^{i-j} \frac{\mu}{n-j-r+1},
$$

that is, the best function to predict $T$ from $X_{j: n}=x$ is a linear function of $x$ (actually, it is the regression line). Then, in this case, the correlation coefficient is more important since it is equal to the regression coefficient and it can be used to measure how accurate these predictions will be. This procedure is illustrated in Example 4.2 below. This property is not necessarily true when the components have a common distribution different than the exponential distribution.

The following example illustrates the definitions and claims above.

Example 2.1. Let $X_{1}, X_{2}, X_{3}$, and $X_{4}$ represent the i.i.d. lifetimes of four components. Let $T_{1}$ and $T_{2}$ be the lifetimes of the following coherent systems with a single shared component: $T_{1}=$ $X_{2: 3}=\min \left(\max \left(X_{1}, X_{2}\right), \max \left(X_{1}, X_{3}\right), \max \left(X_{2}, X_{3}\right)\right)$ and $T_{2}=\min \left(X_{3}, X_{4}\right)$. We may then calculate the probability distribution of the random pair $\left(I_{1}, I_{2}\right)$ by using the permutations given in Table 1. From the above, one may identify the BSM as

$$
\boldsymbol{P}=\left(\begin{array}{cccc}
0 & 0 & 0 & 0 \\
\frac{1}{6} & \frac{1}{6} & \frac{1}{6} & 0 \\
\frac{1}{3} & \frac{1}{6} & 0 & 0 \\
0 & 0 & 0 & 0
\end{array}\right)
$$

From the matrix $\boldsymbol{P}$, which is the equivalent of the bivariate probability mass function of $\left(I_{1}, I_{2}\right)$, we may identify the marginal probability mass function of $I_{1}$ as $\left(0, \frac{1}{2}, \frac{1}{2}, 0\right)$ and that of $I_{2}$ as $\left(\frac{1}{2}, \frac{1}{3}, \frac{1}{6}, 0\right)$. These values coincide with the signatures of order 4 obtained in Table 1 (lines 6 and 2 , respectively) of [29].

In practice, an interesting instance of the occurrence of systems sharing components is when $T_{1}=\psi_{1}\left(X_{1}, \ldots, X_{n}\right)$ and $T_{2}=X_{i}$ for $1 \leq i \leq n$. In this case, the dependence between $T_{1}$

TABLE 1: Calculation of the BSM for the systems in Example 2.1.

\begin{tabular}{cccc}
\hline Equiprobable orderings & $\left(I_{1}, I_{2}\right)$ & Equiprobable orderings & $\left(I_{1}, I_{2}\right)$ \\
\hline$X_{1}<X_{2}<X_{3}<X_{4}$ & $(2,3)$ & $X_{3}<X_{1}<X_{2}<X_{4}$ & $(2,1)$ \\
$X_{1}<X_{2}<X_{4}<X_{3}$ & $(2,3)$ & $X_{3}<X_{1}<X_{4}<X_{2}$ & $(2,1)$ \\
$X_{1}<X_{3}<X_{2}<X_{4}$ & $(2,2)$ & $X_{3}<X_{2}<X_{1}<X_{4}$ & $(2,1)$ \\
$X_{1}<X_{3}<X_{4}<X_{2}$ & $(2,2)$ & $X_{3}<X_{2}<X_{4}<X_{1}$ & $(2,1)$ \\
$X_{1}<X_{4}<X_{2}<X_{3}$ & $(3,2)$ & $X_{3}<X_{4}<X_{1}<X_{2}$ & $(3,1)$ \\
$X_{1}<X_{4}<X_{3}<X_{2}$ & $(3,2)$ & $X_{3}<X_{4}<X_{2}<X_{1}$ & $(3,1)$ \\
$X_{2}<X_{1}<X_{3}<X_{4}$ & $(2,3)$ & $X_{4}<X_{1}<X_{2}<X_{3}$ & $(3,1)$ \\
$X_{2}<X_{1}<X_{4}<X_{3}$ & $(2,3)$ & $X_{4}<X_{1}<X_{3}<X_{2}$ & $(3,1)$ \\
$X_{2}<X_{3}<X_{1}<X_{4}$ & $(2,2)$ & $X_{4}<X_{2}<X_{1}<X_{3}$ & $(3,1)$ \\
$X_{2}<X_{3}<X_{4}<X_{1}$ & $(2,2)$ & $X_{4}<X_{2}<X_{3}<X_{1}$ & $(3,1)$ \\
$X_{2}<X_{4}<X_{1}<X_{3}$ & $(3,2)$ & $X_{4}<X_{3}<X_{1}<X_{2}$ & $(3,1)$ \\
$X_{2}<X_{4}<X_{3}<X_{1}$ & $(3,2)$ & $X_{4}<X_{3}<X_{2}<X_{1}$ & $(3,1)$ \\
\hline
\end{tabular}


and $X_{i}$ can be used to measure the influence of the $i$ th component on the system. This procedure is illustrated in Example 4.3 below. In that example, the main component under this criteria (i.e. that with the lifetime most highly correlated with the system lifetime) coincides with the main component under the Birnbaum importance measure criteria. This case might be used to predict $T_{1}$ when we know that the failure of the $i$ th component occurs at time $t$. Note that the conditional distribution of $\left(T_{1} \mid X_{i}=t\right)$ is a mixture of a discrete distribution (degenerate at $t$ ) with weight $d=\sum_{i=1}^{n} p_{i, i}$ and an absolutely continuous distribution with weight $1-d$ (see Example 4.3 below).

The representations above can be extended to the case of coherent systems with exchangeable components (i.e. its joint distribution is invariant under permutations). Thus, if two coherent systems have lifetimes $T_{1}=\psi_{1}\left(Y_{1}, \ldots, Y_{n_{1}}\right)$ and $T_{2}=\psi_{2}\left(Z_{1}, \ldots, Z_{n_{2}}\right)$, where $\left\{Y_{1}, \ldots, Y_{n_{1}}\right\}$ and $\left\{Z_{1}, \ldots, Z_{n_{2}}\right\}$ are subsets of $\left\{X_{1}, \ldots, X_{n}\right\}$, they share $n_{1,2}>0$ components, $n=n_{1}+$ $n_{2}-n_{1,2}$, and the random vector $\left(X_{1}, \ldots, X_{n}\right)$ has a joint exchangeable absolutely continuous distribution, then the mixture representation (2.3) holds. However, in this case, $F_{i, j: n}$ cannot be computed from (2.4). All the preceding results hold for this case except, of course, those which assume that the components have i.i.d. exponential distributions.

Finally, we should mention that these results can be extended to higher dimensions. Thus, for example, if $T_{1}, T_{2}$, and $T_{3}$ are the lifetimes of three coherent systems based on component lifetimes included in $\left\{X_{1}, \ldots, X_{n}\right\}$, where $X_{1}, \ldots, X_{n}$ are i.i.d. with a common continuous distribution, then the joint distribution $G$ of $\left(T_{1}, T_{2}, T_{3}\right)$ can be written as

$$
G\left(t_{1}, t_{2}, t_{3}\right)=\sum_{i, j, k} p_{i, j, k} F_{i, j, k: n}\left(t_{1}, t_{2}, t_{3}\right),
$$

where $p_{i, j, k}=\mathbb{P}\left(T_{1}=X_{i: n}, T_{2}=X_{j: n}, T_{3}=X_{k: n}\right)$ and $F_{i, j, k: n}$ is the joint distribution of $\left(X_{i: n}, X_{j: n}, X_{k: n}\right)$. The three-dimensional matrix $\boldsymbol{P}=\left(p_{i, j, k}\right)$ is called the trivariate signature matrix.

\section{Ordering results based on bivariate signatures}

In this section, we study how to use the BSM to perform stochastic comparisons. We start with the notion of stochastic precedence. Arcones et al. [1] introduced the concept of stochastic precedence (SP) as an alternative approach to the notion that one random variable is smaller than another. More specifically, if $X$ and $Y$ are two random variables (over the same probability space), then $X$ is smaller than $Y$ in stochastic precedence when $\mathbb{P}(X>Y) \leq \mathbb{P}(X<Y)$. If the random variables $X$ and $Y$ are independent and $X \leq_{\text {st }} Y$, where ' $\leq_{\text {st }}$ ' denotes the usual stochastic order (i.e. $\mathbb{P}(X>t) \leq \mathbb{P}(Y>t)$ for all $t$ ), then $X$ is smaller than $Y$ in the SP sense (see [1]). Hence, stochastic precedence is a necessary property to have the usual stochastic order.

Recently, Hollander and Samaniego [15] (see also [31]) proposed using SP to compare the lifetimes $T_{1}$ and $T_{2}$ of two independent coherent systems. Hollander and Samaniego [15] obtained some expressions to compute $\mathbb{P}\left(T_{1} \leq T_{2}\right)$ based on the signature vectors of both systems in the i.i.d. case. Therefore, these comparisons depend only on the signature vectors and, hence, they are distribution free. Hollander and Samaniego [15] (see also Theorem 5.6 of [31]) obtained similar expressions when the components in both systems are i.i.d. with distributions satisfying the proportional hazard rate (PHR) model, showing that these comparisons do not depend on the baseline hazard rate function. Alternative expressions were obtained in [23], also for the case of independent systems. 
The bivariate signature can be used to perform SP comparisons of coherent systems sharing some (or all) components. Thus, if $\boldsymbol{P}=\left(p_{i, j}\right)$ is the BSM of two coherent systems with lifetimes $T_{1}$ and $T_{2}$, whose respective component lifetimes $\left\{Y_{1}, \ldots, Y_{n_{1}}\right\}$ and $\left\{Z_{1}, \ldots, Z_{n_{2}}\right\}$ are subsets of $\left\{X_{1}, \ldots, X_{n}\right\}$ and $X_{1}, \ldots, X_{n}$ are i.i.d. (respectively exchangeable) with a common (respectively joint absolutely) continuous distribution, then $\mathbb{P}\left(T_{1} \leq T_{2}\right)$ can be computed as

$$
\mathbb{P}\left(T_{1} \leq T_{2}\right)=\sum_{i=1}^{n} \sum_{j=1}^{n} p_{i, j} \mathbb{P}\left(T_{1} \leq T_{2} \mid T_{1}=X_{i: n}, T_{2}=X_{j: n}\right)=\sum_{i \leq j} p_{i, j}
$$

This expression proves that these comparisons are distribution free. Also, note that this method can be applied when $\left\{Y_{1}, \ldots, Y_{n_{1}}\right\} \cap\left\{Z_{1}, \ldots, Z_{n_{2}}\right\}=\varnothing$, that is, when the systems are independent. Hence, it is an alternative method to that proposed in the literature mentioned above (see Examples 4.1 and 4.3 below).

Next, we extend to the bivariate case the ordering results for the stochastic order based on the univariate signature given in Kochar et al. [17] and Navarro et al. [29]. Specifically, they proved (under different assumptions) that if the signature vectors of two coherent systems are ST ordered (as discrete distributions), then so are the system lifetimes. The univariate stochastic order is extended to the multivariate case as follows (see [33, p. 266]).

Definition 3.1. Let $\boldsymbol{X}$ and $\boldsymbol{Y}$ be two $n$-dimensional random vectors. Then we say that $\boldsymbol{X} \leq_{\text {st }} \boldsymbol{Y}$ if $\mathbb{E}(\phi(X)) \leq \mathbb{E}(\phi(\boldsymbol{Y}))$ for all increasing real-valued functions $\phi$ for which these expectations exist.

It is well known that if two random vectors are ST ordered then they are ordered in the lower and upper orthant orders (see [33, p. 308]), that is, if $\boldsymbol{X} \leq_{\text {st }} \boldsymbol{Y}$ then

$$
\mathbb{P}\left(X_{1} \leq x_{1}, \ldots, X_{n} \leq x_{n}\right) \geq \mathbb{P}\left(Y_{1} \leq x_{1}, \ldots, Y_{n} \leq x_{n}\right)
$$

and

$$
\mathbb{P}\left(X_{1}>x_{1}, \ldots, X_{n}>x_{n}\right) \leq \mathbb{P}\left(Y_{1}>x_{1}, \ldots, Y_{n}>x_{n}\right)
$$

for all $x_{1}, \ldots, x_{n}$.

Now we can extend the univariate results for the stochastic order as follows.

Theorem 3.1. Let $T_{1}$ and $T_{2}$ be the lifetimes of two coherent systems whose respective component lifetimes $\left\{Y_{1}, \ldots, Y_{n_{1}}\right\}$ and $\left\{Z_{1}, \ldots, Z_{n_{2}}\right\}$ are subsets of $\left\{X_{1}, \ldots, X_{n}\right\}$, and $X_{1}, \ldots, X_{n}$ are i.i.d. with a common continuous distribution $F$. Let $I$ be the random vector defined by (2.1) for $\left(T_{1}, T_{2}\right)$. Let $T_{1}^{*}$ and $T_{2}^{*}$ be the lifetimes of two coherent systems whose respective component lifetimes $\left\{Y_{1}^{*}, \ldots, Y_{n_{1}^{*}}^{*}\right\}$ and $\left\{Z_{1}^{*}, \ldots, Z_{n_{2}^{*}}^{*}\right\}$ are subsets of $\left\{X_{1}^{*}, \ldots, X_{n}^{*}\right\}$, and $X_{1}^{*}, \ldots, X_{n}^{*}$ are i.i.d. with a common continuous distribution $F^{*}$. Let $\boldsymbol{I}^{*}$ be the random vector defined by (2.1) for $\left(T_{1}^{*}, T_{2}^{*}\right)$. If $\boldsymbol{I} \leq_{\mathrm{st}} \boldsymbol{I}^{*}$ and $X_{1} \leq_{\mathrm{st}} X_{1}^{*}$, then

$$
\left(T_{1}, T_{2}\right) \leq_{\mathrm{st}}\left(T_{1}^{*}, T_{2}^{*}\right) .
$$

Proof. From (2.3) we have

$$
\mathbb{E}\left(\phi\left(T_{1}, T_{2}\right)\right)=\int_{\mathbb{R}^{2}} \phi\left(t_{1}, t_{2}\right) \mathrm{d} G\left(t_{1}, t_{2}\right)=\sum_{i=1}^{n} \sum_{j=1}^{n} p_{i, j} \mathbb{E}\left(\phi\left(X_{i: n}, X_{j: n}\right)\right),
$$


where $\boldsymbol{P}=\left(p_{i, j}\right)$ is the BSM of $\left(T_{1}, T_{2}\right)$. Analogously,

$$
\mathbb{E}\left(\phi\left(T_{1}^{*}, T_{2}^{*}\right)\right)=\int_{\mathbb{R}^{2}} \phi\left(t_{1}, t_{2}\right) \mathrm{d} G^{*}\left(t_{1}, t_{2}\right)=\sum_{i=1}^{n} \sum_{j=1}^{n} p_{i, j}^{*} \mathbb{E}\left(\phi\left(X_{i: n}^{*}, X_{j: n}^{*}\right)\right),
$$

where $\boldsymbol{P}^{*}=\left(p_{i, j}^{*}\right)$ is the bivariate signature of $\left(T_{1}^{*}, T_{2}^{*}\right)$.

Let $k \geq i$. Then $X_{i: n} \leq X_{k: n}$, and, as $\phi$ is increasing, we have $\phi\left(X_{i: n}, X_{j: n}\right) \leq \phi\left(X_{k: n}, X_{j: n}\right)$ and $\mathbb{E}\left(\phi\left(X_{i: n}, X_{j: n}\right)\right) \leq \mathbb{E}\left(\phi\left(X_{k: n}, X_{j: n}\right)\right)$. A similar property holds for $k \geq j$. Therefore, the function $q(i, j)=\mathbb{E}\left(\phi\left(X_{i: n}, X_{j: n}\right)\right)$ is increasing in $i$ and $j$.

On the other hand, using the fact that $X_{1} \leq_{\text {st }} X_{1}^{*}$, from Theorem 6.B.16(b) of [33, p. 273], we have $\left(X_{1}, \ldots, X_{n}\right) \leq_{\text {st }}\left(X_{1}^{*}, \ldots, X_{n}^{*}\right)$. Hence, since $\Psi\left(x_{1}, \ldots, x_{n}\right)=\phi\left(x_{i: n}, x_{j: n}\right)$ is an increasing function, we have

$$
q(i, j)=\mathbb{E}\left(\phi\left(X_{i: n}, X_{j: n}\right)\right) \leq \mathbb{E}\left(\phi\left(X_{i: n}^{*}, X_{j: n}^{*}\right)\right)=q^{*}(i, j) .
$$

Therefore,

$$
\begin{aligned}
\mathbb{E}\left(\phi\left(T_{1}, T_{2}\right)\right) & =\sum_{i=1}^{n} \sum_{j=1}^{n} p_{i, j} q(i, j) \\
& \leq \sum_{i=1}^{n} \sum_{j=1}^{n} p_{i, j}^{*} q(i, j) \quad\left(\boldsymbol{I} \leq_{\mathrm{st}} \boldsymbol{I}^{*} \text { and } q\right. \text { is increasing) } \\
& \leq \sum_{i=1}^{n} \sum_{j=1}^{n} p_{i, j}^{*} q^{*}(i, j) \quad\left(\text { from }(3.5) \text { and } p_{i, j}^{*} \geq 0\right) \\
& =\mathbb{E}\left(\phi\left(T_{1}^{*}, T_{2}^{*}\right)\right)
\end{aligned}
$$

for any increasing function $\phi$ for which these expectations exist. Then (3.4) holds.

A similar result can be obtained for systems with exchangeable components.

Theorem 3.2. Let $T_{1}$ and $T_{2}$ be the lifetimes of two coherent systems whose respective component lifetimes $\left\{Y_{1}, \ldots, Y_{n_{1}}\right\}$ and $\left\{Z_{1}, \ldots, Z_{n_{2}}\right\}$ are subsets of $\left\{X_{1}, \ldots, X_{n}\right\}$, and $\left(X_{1}, \ldots, X_{n}\right)$ is an exchangeable random vector with a joint absolutely continuous distribution. Let $I$ be the random vector defined by (2.1). Let $T_{1}^{*}$ and $T_{2}^{*}$ be the lifetimes of two coherent systems whose respective component lifetimes $\left\{Y_{1}^{*}, \ldots, Y_{n_{1}^{*}}^{*}\right\}$ and $\left\{Z_{1}^{*}, \ldots, Z_{n_{2}^{*}}^{*}\right\}$ are subsets of $\left\{X_{1}^{*}, \ldots, X_{n}^{*}\right\}$, and $\left(X_{1}^{*}, \ldots, X_{n}^{*}\right)$ is an exchangeable random vector with a joint absolutely continuous distribution. Let $\boldsymbol{I}^{*}$ be the random vector defined by $(2.1)$ for $\left(T_{1}^{*}, T_{2}^{*}\right)$. If $\boldsymbol{I} \leq_{\mathrm{st}} \boldsymbol{I}^{*}$ and $\left(X_{1}, \ldots, X_{n}\right) \leq_{\text {st }}\left(X_{1}^{*}, \ldots, X_{n}^{*}\right)$, then $\left(T_{1}, T_{2}\right) \leq_{\text {st }}\left(T_{1}^{*}, T_{2}^{*}\right)$.

The proof is analogous to the proof of the i.i.d. case. Note that both theorems can be applied to coherent systems having the same components $\left(X_{1}, \ldots, X_{n}\right)$. In this case, we only need the condition $\boldsymbol{I} \leq_{\mathrm{st}} \boldsymbol{I}^{*}$. Next we analyze the conditions on the bivariate signatures $\boldsymbol{P}$ and $\boldsymbol{P}^{*}$ to have $\boldsymbol{I} \leq_{\mathrm{st}} \boldsymbol{I}^{*}$. First, we define the following matrix ordering.

Definition 3.2. Let $\boldsymbol{A}=\left(a_{i, j}\right)$ and $\boldsymbol{A}^{*}=\left(a_{i, j}^{*}\right)$ be two $n \times m$ matrices with the same total mass, that is, with $\sum_{i=1}^{n} \sum_{j=1}^{m} a_{i, j}=\sum_{i=1}^{n} \sum_{j=1}^{m} a_{i, j}^{*}$. Then we say that $\boldsymbol{A}$ is less than $\boldsymbol{A}^{*}$ in the south-east shift order (written as $A \leq S / E \rightarrow A^{*}$ ) if $\boldsymbol{A}^{*}$ can be obtained from $\boldsymbol{A}$ through a finite sequence of transformations in which a positive mass $c>0$ is moved from the term $a_{i, j}$ to the term $a_{r, s}$ with $r \geq i$ and $s \geq j$ (i.e. the new terms are $a_{i, j}-c$ and $a_{r, s}+c$, respectively). 
For example, the following matrices are $S / E \rightarrow$ ordered:

$$
\left(\begin{array}{ccc}
0 & \frac{2}{3} & \frac{1}{3} \\
0 & 0 & 0 \\
0 & 0 & 0
\end{array}\right) \rightarrow\left(\begin{array}{ccc}
0 & \frac{1}{6} & \frac{1}{3} \\
0 & \frac{1}{2} & 0 \\
0 & 0 & 0
\end{array}\right) \rightarrow\left(\begin{array}{ccc}
0 & \frac{1}{6} & \frac{1}{6} \\
0 & \frac{1}{2} & \frac{1}{6} \\
0 & 0 & 0
\end{array}\right) \rightarrow\left(\begin{array}{ccc}
0 & \frac{1}{6} & \frac{1}{6} \\
0 & \frac{1}{6} & \frac{1}{2} \\
0 & 0 & 0
\end{array}\right)
$$

In the next result we use this matrix order to characterize $\boldsymbol{I} \leq_{\mathrm{st}} \boldsymbol{I}^{*}$ using the bivariate signatures.

Theorem 3.3. Let $\boldsymbol{I}$ be the random vector defined by $\boldsymbol{I}=(i, j)$ with probability $p_{i, j} \geq 0$ for $i, j=1, \ldots, n$. Let $\boldsymbol{I}^{*}$ be the random vector defined by $\boldsymbol{I}^{*}=(i, j)$ with probability $p_{i, j}^{*} \geq 0$ for $i, j=1, \ldots, n$. Let $\boldsymbol{P}=\left(p_{i, j}\right)$ and $\boldsymbol{P}^{*}=\left(p_{i, j}^{*}\right)$. Then $\boldsymbol{I} \leq_{\mathrm{st}} \boldsymbol{I}^{*}$ is equivalent to $\boldsymbol{P} \leq S / E \rightarrow \boldsymbol{P}^{*}$.

Proof. Let us assume that $\boldsymbol{P} \leq S / E \rightarrow \boldsymbol{P}^{*}$, that is, there exists a finite sequence of matrices $\boldsymbol{A}_{1}, \ldots, \boldsymbol{A}_{k}$ as those described in Definition 3.2 such that

$$
\boldsymbol{P}=\boldsymbol{A}_{1} \rightarrow \boldsymbol{A}_{2} \rightarrow \cdots \rightarrow \boldsymbol{A}_{k}=\boldsymbol{P}^{*},
$$

where $\boldsymbol{A}_{l} \rightarrow \boldsymbol{A}_{l+1}$ means that $\boldsymbol{A}_{l+1}$ is obtained from $\boldsymbol{A}_{l}$ by a translation of a positive mass $c_{l}>0$ from the $(i, j)$ th term of $\boldsymbol{A}_{l}$ to the $(r, s)$ th term of $\boldsymbol{A}_{l}$ with $r \geq i$ and $s \geq j$.

Let $\phi(i, j)$ be an increasing function. As $\boldsymbol{I}$ and $\boldsymbol{I}^{*}$ have a finite support, without loss of generality, we can assume that $\phi(i, j) \geq 0$ (if it takes negative values then we can replace $\phi(i, j)$ by $\left.\phi(i, j)-\min _{r, s=1, \ldots, n} \phi(r, s)\right)$. Hence, if $\boldsymbol{A}_{l}=\left(a_{i, j}^{(l)}\right)$ then

$$
\begin{aligned}
\phi(i, j) a_{i, j}^{(l)}+\phi(r, s) a_{r, s}^{(l)} & =\phi(i, j)\left(a_{i, j}^{(l)}-c_{l}+c_{l}\right)+\phi(r, s) a_{r, s}^{(l)} \\
& =\phi(i, j)\left(a_{i, j}^{(l)}-c_{l}\right)+\phi(i, j) c_{l}+\phi(r, s) a_{r, s}^{(l)} \\
& \leq \phi(i, j)\left(a_{i, j}^{(l)}-c_{l}\right)+\phi(r, s)\left(a_{r, s}^{(l)}+c_{l}\right) \\
& =\phi(i, j) a_{i, j}^{(l+1)}+\phi(r, s) a_{r, s}^{(l+1)},
\end{aligned}
$$

where, to obtain the inequality, we used the facts that $\phi$ is increasing and $c_{l}>0$. Therefore,

$$
\sum_{i=1}^{n} \sum_{j=1}^{n} \phi(i, j) a_{i, j}^{(l)} \leq \sum_{i=1}^{n} \sum_{j=1}^{n} \phi(i, j) a_{i, j}^{(l+1)}
$$

for $l=1, \ldots, k-1$, and then

$$
\sum_{i=1}^{n} \sum_{j=1}^{n} \phi(i, j) p_{i, j} \leq \sum_{i=1}^{n} \sum_{j=1}^{n} \phi(i, j) p_{i, j}^{*}
$$

for any increasing function $\phi$. Hence, $\boldsymbol{I} \leq_{\mathrm{st}} \boldsymbol{I}^{*}$.

Conversely, let us assume that $\boldsymbol{I} \leq_{\mathrm{st}} \boldsymbol{I}^{*}$ and, for $i, j \in\{1, \ldots, n\}$, let us consider the function $\phi$ defined by $\phi(r, s)=1$ for $r \geq i$ and $s \geq j$, and 0 elsewhere. Then we have

$$
\sum_{r=i}^{n} \sum_{s=j}^{n} p_{i, j} \leq \sum_{r=i}^{n} \sum_{s=j}^{n} p_{i, j}^{*}
$$

for all $i, j$. Hence, using the fact that $\sum_{i=1}^{n} \sum_{j=1}^{n} p_{i, j}=\sum_{i=1}^{n} \sum_{j=1}^{n} p_{i, j}=1, \boldsymbol{P}^{*}$ can be obtained by a finite sequence of transformations as described in Definition 3.2. 
The preceding result is used in Example 4.4 below to obtain the bivariate stochastic order between systems. Finally, note that, under the assumptions of Theorem 3.1, if $\boldsymbol{P} \leq S / E \rightarrow \boldsymbol{P}^{*}$ then, from (3.2) and (3.3), the series and parallel systems obtained from $\left(T_{1}, T_{2}\right)$ and $\left(T_{1}^{*}, T_{2}^{*}\right)$ are stochastically ordered, that is, $\min \left(T_{1}, T_{2}\right) \leq_{\mathrm{st}} \min \left(T_{1}^{*}, T_{2}^{*}\right)$ and $\max \left(T_{1}, T_{2}\right) \leq_{\mathrm{st}}$ $\max \left(T_{1}^{*}, T_{2}^{*}\right)$.

\section{Examples}

In this section, we present some examples to illustrate the applications of bivariate signatures. In the first example we show how to compute the bivariate signature of two coherent systems. This example shows that the bivariate signature might depend on where we place the components in a system structure, that is, the precise labeling of the components.

Example 4.1. Let us consider two coherent systems with the same i.i.d. components and respective system lifetimes $T_{1}=\min \left(X_{1}, \max \left(X_{2}, X_{3}\right)\right)$ and $T_{2}=\max \left(X_{1}, \min \left(X_{2}, X_{3}\right)\right)$. Then the $3 !=6$ permutations lead to the results given in Table 2 . Hence, the bivariate signature of $\left(T_{1}, T_{2}\right)$ is

$$
\boldsymbol{P}=\left(\begin{array}{lll}
0 & \frac{1}{3} & 0 \\
0 & \frac{1}{3} & \frac{1}{3} \\
0 & 0 & 0
\end{array}\right)
$$

and their joint distribution can be written as

$$
G\left(t_{1}, t_{2}\right)=\frac{1}{3} F_{1,2: 3}\left(t_{1}, t_{2}\right)+\frac{1}{3} F_{2,3: 3}\left(t_{1}, t_{2}\right)+\frac{1}{3} F_{2: 3}\left(\min \left(t_{1}, t_{2}\right)\right) .
$$

Note that $G$ is not absolutely continuous since $\mathbb{P}\left(T_{1}=T_{2}\right)=p_{2,2}=\frac{1}{3}$. Moreover, $\mathbb{P}\left(T_{1} \leq T_{2}\right)$ can be computed as $\mathbb{P}\left(T_{1} \leq T_{2}\right)=\sum_{i \leq j} p_{i, j}=1$. Therefore, $T_{1} \leq_{\mathrm{st}} T_{2}$. Actually, since $T_{1} \leq T_{2}$, then we have $T_{1} \leq_{\mathrm{st}} T_{2}$. Also, note that the (univariate) signatures of $T_{1}$ and $T_{2}$ can be obtained as the marginal distributions of $\boldsymbol{P}$ (i.e. adding rows or columns), obtaining $\left(\frac{1}{3}, \frac{2}{3}, 0\right)$ and $\left(0, \frac{2}{3}, \frac{1}{3}\right)$, respectively.

The coherent systems with i.i.d. components and lifetimes $T_{2}=\max \left(X_{1}, \min \left(X_{2}, X_{3}\right)\right)$ and $T_{2}^{*}=\max \left(X_{2}, \min \left(X_{1}, X_{3}\right)\right)$ have the same signature. However, the bivariate signature of $T_{1}$ and $T_{2}^{*}$ is

$$
\boldsymbol{P}^{*}=\left(\begin{array}{ccc}
0 & \frac{1}{6} & \frac{1}{6} \\
0 & \frac{1}{2} & \frac{1}{6} \\
0 & 0 & 0
\end{array}\right),
$$

which is not equal to $\boldsymbol{P}$. Moreover, $\mathbb{P}\left(T_{1}=T_{2}^{*}\right)=p_{2,2}^{*}=\frac{1}{2} \neq \frac{1}{3}=p_{2,2}$.

TABle 2.

\begin{tabular}{cccc}
\hline Equiprobable orderings & $T_{1}$ & $T_{2}$ & $\boldsymbol{I}$ \\
\hline$X_{1}<X_{2}<X_{3}$ & $X_{1}=X_{1: 3}$ & $X_{2}=X_{2: 3}$ & $(1,2)$ \\
$X_{1}<X_{3}<X_{2}$ & $X_{1}=X_{1: 3}$ & $X_{3}=X_{2: 3}$ & $(1,2)$ \\
$X_{2}<X_{1}<X_{3}$ & $X_{1}=X_{2: 3}$ & $X_{1}=X_{2: 3}$ & $(2,2)$ \\
$X_{2}<X_{3}<X_{1}$ & $X_{3}=X_{2: 3}$ & $X_{1}=X_{3: 3}$ & $(2,3)$ \\
$X_{3}<X_{1}<X_{2}$ & $X_{1}=X_{2: 3}$ & $X_{1}=X_{2: 3}$ & $(2,2)$ \\
$X_{3}<X_{2}<X_{1}$ & $X_{2}=X_{2: 3}$ & $X_{1}=X_{3: 3}$ & $(2,3)$ \\
\hline
\end{tabular}


The next example shows how these computations can be simplified when one system is a $k$-out-of- $n$ system.

Example 4.2. Let us consider $T_{1}=X_{1: 3}$ and $T_{2}=\max \left(X_{1}, \min \left(X_{2}, X_{3}\right)\right)$ with signatures $(1,0,0)$ and $\left(0, \frac{2}{3}, \frac{1}{3}\right)$, respectively. Hence, their bivariate signature is

$$
\boldsymbol{P}=\left(\begin{array}{ccc}
0 & \frac{2}{3} & \frac{1}{3} \\
0 & 0 & 0 \\
0 & 0 & 0
\end{array}\right)
$$

and their joint distribution can be written as

$$
G\left(t_{1}, t_{2}\right)=\frac{2}{3} F_{1,2: 3}\left(t_{1}, t_{2}\right)+\frac{1}{3} F_{1,3: 3}\left(t_{1}, t_{2}\right) .
$$

Note that if $X_{1}, X_{2}$, and $X_{3}$ are i.i.d. with a common absolutely continuous distribution, then $G$ is absolutely continuous since $\mathbb{P}\left(T_{1}=T_{2}\right)=0$.

The covariance between $X_{1: 3}$ and $T_{2}$ can be computed as

$$
\operatorname{cov}\left(X_{1: 3}, T_{2}\right)=\frac{2}{3} \sigma_{1,2: 3}+\frac{1}{3} \sigma_{1,3: 3}
$$

If the component lifetimes have exponential distributions then $\sigma_{1,2: 3}=\sigma_{1,3: 3}=\sigma_{1,1: 3}$ and we have

$$
\operatorname{cov}\left(X_{1: 3}, T_{2}\right)=\sigma_{1,1: 3}=\operatorname{var}\left(X_{1: 3}\right)=\frac{1}{9} \mu^{2} .
$$

Hence,

$$
\operatorname{corr}\left(X_{1: 3}, T_{2}\right)=\sqrt{\frac{\operatorname{var}\left(X_{1: 3}\right)}{\operatorname{var}\left(T_{2}\right)}} .
$$

Then, since $\operatorname{var}\left(T_{2}\right)=\frac{11}{12} \mu^{2}$, we have

$$
\operatorname{corr}\left(X_{1: 3}, T_{2}\right)=\sqrt{\frac{1 / 9}{11 / 12}}=\frac{2}{\sqrt{33}}=0.34816
$$

and $\operatorname{corr}^{2}\left(X_{1: 3}, T_{2}\right)=\frac{4}{33}=0.12121$, that is, a linear function of $X_{1: 3}$ only explains $12.12 \%$ of the variation in $T_{2}$. From (2.5), to predict $T_{2}$, we should use

$$
\mathbb{E}\left(T_{2} \mid X_{1: 3}=x\right)=x+\frac{2}{3} \mu_{1: 2}+\frac{1}{3} \mu_{2: 2}=x+\frac{2}{3} \frac{1}{2} \mu+\frac{1}{3} \frac{3}{2} \mu=x+\frac{5}{6} \mu .
$$

For example, if $\mu=1$ then the regression curve (line) is $\mathbb{E}\left(T_{2} \mid X_{1: 3}=x\right)=0.83333+x$. If $\mu$ is unknown then we might use $\mathbb{E}\left(X_{1: 3}\right)=\mu / 3$ to estimate $\mu$ as $\widehat{\mu}=3 x$. Then we get $\mathbb{E}\left(T_{2} \mid X_{1: 3}=x\right) \simeq 7 x / 2$.

The next example shows that these representations can also be applied to systems sharing some components (not necessarily all of them). Specifically, we consider the case $T_{2}=X_{i}$ for $i=1,2,3$.

Example 4.3. Let us consider the systems with lifetimes $T_{1}=\min \left(X_{1}, \max \left(X_{2}, X_{3}\right)\right)$ and $T_{2}=X_{i}$ for $i=1,2,3$. In this case, the dependence between $T_{1}$ and $X_{i}$ can be used to measure the relevance of the $i$ th component in the system. Hence, from Table 2 we have the bivariate signature of $\left(T_{1}, X_{1}\right)$ to be

$$
\boldsymbol{P}_{1}=\boldsymbol{P}\left(T_{1}, X_{1}\right)=\left(\begin{array}{ccc}
\frac{1}{3} & 0 & 0 \\
0 & \frac{1}{3} & \frac{1}{3} \\
0 & 0 & 0
\end{array}\right)
$$


and their joint distribution can be written as

$$
G\left(t_{1}, t_{2}\right)=\frac{1}{3} F_{1: 3}\left(\min \left(t_{1}, t_{2}\right)\right)+\frac{1}{3} F_{2: 3}\left(\min \left(t_{1}, t_{2}\right)\right)+\frac{1}{3} F_{2,3: 3}\left(t_{1}, t_{2}\right) .
$$

Note that the signature of order 3 of $X_{1}$ (obtained by adding the rows) is $\left(\frac{1}{3}, \frac{1}{3}, \frac{1}{3}\right)$, that is, $X_{1}$ is a uniform mixture of $X_{1: 3}, X_{2: 3}$, and $X_{3: 3}$. Analogously, we have the bivariate signature of $\left(T_{1}, X_{i}\right)$ to be

$$
\boldsymbol{P}_{i}=\boldsymbol{P}\left(T_{1}, X_{i}\right)=\left(\begin{array}{ccc}
0 & \frac{1}{6} & \frac{1}{6} \\
\frac{1}{3} & \frac{1}{6} & \frac{1}{6} \\
0 & 0 & 0
\end{array}\right)
$$

for $i=2,3$. Note that the signature of order 3 of $X_{i}$ is also $\left(\frac{1}{3}, \frac{1}{3}, \frac{1}{3}\right)$ for $i=2,3$.

Then, for $x>0$, the conditional distribution of $\left(T_{1} \mid X_{1}=x\right)$ is given by

$$
G_{T_{1} \mid 1}(t \mid x)=\frac{2}{3} D_{x}(t)+\frac{1}{3} G_{2: 2}(t \mid x),
$$

where $D_{x}(t)=0$ (or $D_{x}(t)=1$ ) for $t<x$ (or $t \geq x$ ) is the distribution function of a degenerate random variable at $x$ and $G_{2: 2}(t \mid x)=\bar{F}^{2}(t) / F^{2}(x)$ (or $G_{2: 2}(t \mid x)=1$ ) for $t<x$ (or $t \geq x$ ) is the distribution of $Y_{2: 2}$, where $Y_{1}$ and $Y_{2}$ are i.i.d. with common distribution function $\mathbb{P}\left(Y_{j} \leq t\right)=F(t) / F(x)$ (or $\mathbb{P}\left(Y_{j} \leq t\right)=1$ ) for $t<x$ (or $t \geq x$ ) and $j=1,2$. Note that this last distribution is the distribution of the right-truncated variable $\left(X_{i} \mid X_{i}<x\right)$. Similar results can be obtained for $\left(T_{1} \mid X_{i}=x\right), i=2,3$.

In particular, if the components have a common exponential distribution with mean $\mu$ then a straightforward calculation shows that

$$
\operatorname{cov}\left(T_{1}, X_{1}\right)=\frac{7}{18} \mu^{2}=0.38889 \mu^{2} .
$$

Hence, using the facts that $\operatorname{var}\left(T_{1}\right)=\mu^{2} / 3$ and $\operatorname{var}\left(X_{1}\right)=\mu^{2}$, we have

$$
\operatorname{corr}\left(T_{1}, X_{1}\right)=\frac{7 / 18}{\sqrt{1 / 3} \sqrt{1}}=\frac{7}{18} \sqrt{3}=0.67358,
$$

that is, we can explain $45.37 \%$ of the variation of $T_{1}$ by using the regression line based on $X_{1}$. Analogously, for the other components, we have

$$
\operatorname{cov}\left(T_{1}, X_{i}\right)=\frac{5}{36} \mu^{2}=0.13889 \mu^{2}
$$

and

$$
\operatorname{corr}\left(T_{1}, X_{i}\right)=\frac{5 / 36}{\sqrt{1 / 3} \sqrt{1}}=\frac{5}{36} \sqrt{3}=0.24056
$$

for $i=2,3$, that is, we can explain only $5.78 \%$ of the variation of $T_{1}$ by using the regression line based on $X_{i}$ for $i=2,3$. Hence, the most important (i.e. the most highly correlated) component for the system lifetime is the first component. The first component is also the most important component by using the Birnbaum importance measure.

These representations can also be applied to compare the same systems using SP. Thus, we have $\mathbb{P}\left(T_{1} \leq X_{1}\right)=1$ and $\mathbb{P}\left(T_{1} \leq X_{i}\right)=\frac{2}{3}$ for $i=1,2$. Analogously, if we want to compare $T_{1}$ with an independent component $X_{4}$ (with the same distribution as $X_{1}, X_{2}$, and $X_{3}$ ), we first need to compute the bivariate signature of $\left(T_{1}, X_{4}\right)$, obtaining

$$
\boldsymbol{P}_{4}=\boldsymbol{P}\left(T_{1}, X_{4}\right)=\left(\begin{array}{cccc}
0 & \frac{1}{12} & \frac{1}{12} & \frac{1}{12} \\
\frac{1}{12} & 0 & \frac{1}{6} & \frac{1}{6} \\
\frac{1}{6} & \frac{1}{6} & 0 & 0 \\
0 & 0 & 0 & 0
\end{array}\right) .
$$


Note that the respective signatures of order 4 of $T_{1}$ and $X_{4}$ can be computed as the marginals of $\boldsymbol{P}_{4}$, obtaining $\left(\frac{1}{4}, \frac{5}{12}, \frac{1}{3}, 0\right)$ and $\left(\frac{1}{4}, \frac{1}{4}, \frac{1}{4}, \frac{1}{4}\right)$. These coincide with the values given in Table 1 (lines 1 and 5) of [29]. In this case, the joint distribution representation is not of interest since $T_{1}$ and $X_{4}$ are independent. To compare them using SP, from (3.1), we have

$$
\mathbb{P}\left(T_{1} \leq X_{4}\right)=\frac{7}{12}=0.58333,
$$

and, hence, $T_{1}$ stochastically precedes $X_{4}$ (i.e. this system is worse than a single independent component $58.333 \%$ of the time). This value coincides with the value obtained with the calculations presented in [15], [23], and [31, pp. 68-74].

Finally, in the next example, we show how to obtain multivariate stochastic ordering results from Theorems 3.1-3.3.

Example 4.4. Let us consider $T_{1}=X_{1: 3}$ and $T_{2}=\max \left(X_{1}, \min \left(X_{2}, X_{3}\right)\right)$ with bivariate signature

$$
\boldsymbol{P}=\left(\begin{array}{ccc}
0 & \frac{2}{3} & \frac{1}{3} \\
0 & 0 & 0 \\
0 & 0 & 0
\end{array}\right)
$$

(see Example 4.2), and $T_{1}^{*}=\min \left(X_{1}^{*}, \max \left(X_{2}^{*}, X_{3}^{*}\right)\right)$ and $T_{2}^{*}=\max \left(X_{1}^{*}, \min \left(X_{2}^{*}, X_{3}^{*}\right)\right.$ ) with bivariate signature

$$
\boldsymbol{P}^{*}=\left(\begin{array}{ccc}
0 & \frac{1}{6} & \frac{1}{6} \\
0 & \frac{1}{2} & \frac{1}{6} \\
0 & 0 & 0
\end{array}\right)
$$

(see Example 4.1), where $X_{1}, X_{2}$, and $X_{3}$ are i.i.d. with a common absolutely continuous distribution $F$, and $X_{1}^{*}, X_{2}^{*}$, and $X_{3}^{*}$ are i.i.d. with a common absolutely continuous

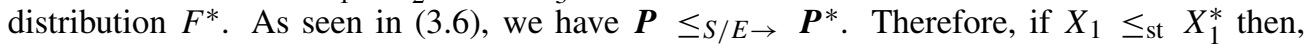
from Theorems 3.1 and 3.3, we have $\left(T_{1}, T_{2}\right) \leq_{\mathrm{st}}\left(T_{1}^{*}, T_{2}^{*}\right)$.

Analogously, if the components are dependent and we assume that $\left(X_{1}, X_{2}, X_{3}\right)$ and $\left(X_{1}^{*}, X_{2}^{*}, X_{3}^{*}\right)$ have absolutely continuous joint distributions and satisfy

$$
\left(X_{1}, X_{2}, X_{3}\right) \leq_{\mathrm{st}}\left(X_{1}^{*}, X_{2}^{*}, X_{3}^{*}\right),
$$

then, from Theorems 3.2 and 3.3, we have $\left(T_{1}, T_{2}\right) \leq_{\text {st }}\left(T_{1}^{*}, T_{2}^{*}\right)$.

\section{Acknowledgements}

We would like to thank the anonymous reviewer for several helpful suggestions. The authors acknowledge financial support for this research from MEC-FEDER and Fundación Séneca (CARM) under grants MTM2009-08311 and 08627/PI/08 (JN), the US Army Research Office W911NF-11-0428 (FJS), and the Natural Sciences and Engineering Research Council of Canada through an Individual Discovery Grant (NB).

\section{References}

[1] Arcones, M. A., Kvam, P. H. And Samaniego, F. J. (2002). Nonparametric estimation of a distribution subject to a stochastic precedence constraint. J. Amer. Statist. Assoc. 97, 170-182.

[2] Asadi, M. AND Goliforushani, S. (2008). On the mean residual life function of coherent systems. IEEE Trans. Reliab. 57, 574-580.

[3] Bairamov, I. AND ARnold, B. C. (2008). On the residual lifelengths of the remaining components in an $n-k+1$-out-of- $n$ system. Statist. Prob. Lett. 78, 945-952.

[4] Balakrishnan, N. (2007). Permanents, order statistics, outliers, and robustness. Rev. Mat. Complut. 20, 7-107. 
[5] Barlow, R. E. and Proschan, F. (1975). Statistical Theory of Reliability and Life Testing. Holt, Rinehart and Winston, New York.

[6] Burkschat, M. and Navarro, J. (2013). Dynamic signatures of coherent systems based on sequential order statistics. J. Appl. Prob. 50, 272-287.

[7] DA, G., Zheng, B. And Hu, T. (2012). On computing signatures of coherent systems. J. Multivariate Anal. 103, $142-150$.

[8] David, H. A. and Nagaraja, H. N. (2003). Order Statistics, 3rd edn. John Wiley, Hoboken, NJ.

[9] Epstein, B. and Sobel, M. (1953). Life testing. J. Amer. Statist. Assoc. 48, 486-502.

[10] Gertsbakh, I. and Shrungin, Y. (2011). Network Reliability and Resilience. Springer, New York.

[11] Gertsbakh, I. and Shpungin, Y. (2012). Multidimensional spectra of multistate systems with binary components. In Recent Advances in System Reliability, eds A. Lisniansky and I. Frenkel, Springer, London, pp. 49-61.

[12] Gertsbakh, I. And Shpungin, Y. (2012). Stochastic models of network survivability. Quality Tech. Quantitative Manag. 9, 45-58.

[13] Gertsbakh, I., Shpungin, Y. and Spizzichino, F. (2012). Two-dimensional signatures. J. Appl. Prob. 49, 416-429.

[14] Goliforushani, S., Asadi, M. and Balakrishnan, N. (2012). On the residual and inactivity times of the components of used coherent systems. J. Appl. Prob. 49, 385-404.

[15] Hollander, M. and Samaniego, F. J. (2008). On comparing the reliability of arbitrary systems via stochastic precedence. In Advances in Mathematical Modeling for Reliability, eds T. Bedford et al., IOS Press, Amsterdam, pp. 129-137.

[16] Khaledi, B.-E. And Shaked, M. (2007). Ordering conditional lifetimes of coherent systems. J. Statist. Planning Infer. 137, 1173-1184.

[17] Kochar, S., MukerJee, H. and Samaniego, F. J. (1999). The "signature" of a coherent system and its application to comparison among systems. Naval Res. Logistics 46, 507-523.

[18] Li, X. and Zhang, Z. (2008). Some stochastic comparisons of conditional coherent systems. Appl. Stoch. Models Business Industry 24, 541-549.

[19] Li, X. AND ZhaO, P. (2008). Stochastic comparison on general inactivity time and general residual life of $k$-out-of- $n$ systems. Commun. Statist. Simul. Comput. 37, 1005-1019.

[20] Marichal, J.-L. And Mathonet, P. (2011). Extensions of system signatures to dependent lifetimes: explicit expressions and interpretations. J. Multivariate Anal. 102, 931-936.

[21] Marichal, J.-L., Mathonet, P. and Waldhauser, T. (2011). On signature-based expressions of system reliability. J. Multivariate Anal. 102, 1410-1416.

[22] Navarro, J. and Balakrishnan, N. (2010). Study of some measures of dependence between order statistics and systems. J. Multivariate Anal. 101, 52-67.

[23] Navarro, J. and Rubio, R. (2010). Comparisons of coherent systems using stochastic precedence. TEST 19, 469-486.

[24] Navarro, J. and Rubio, R. (2011). A note on necessary and sufficient conditions for ordering properties of coherent systems with exchangeable components. Naval Res. Logistics 58, 478-489.

[25] Navarro, J., Balakrishnan, N. and Samaniego, F. J. (2008). Mixture representations of residual lifetimes of used systems. J. Appl. Prob. 45, 1097-1112.

[26] Navarro, J., Samaniego, F. J. and Balakrishnan, N. (2010). The joint signature of coherent systems with shared components. J. Appl. Prob. 47, 235-253.

[27] Navarro, J., Samaniego, F. J. and Balakrishnan, N. (2011). Signature-based representations for the reliability of systems with heterogeneous components. J. Appl. Prob. 48, 856-867.

[28] Navarro, J., Samaniego, F. J. and Balakrishnan, N. (2013). Mixture representations for the joint distribution of two coherent systems with shared components. Tech. Rep. 1-2013, Department of Statitstics and Operational Research, Universidad de Murcia.

[29] Navarro, J., Samaniego, F. J., Balakrishnan, N. and Bhattacharya, D. (2008). On the application and extension of system signatures in engineering reliability. Naval Res. Logistics 55, 313-327.

[30] Samaniego, F. J. (1985). On closure of the IFR class under formation of coherent systems. IEEE Trans. Reliab. R-34, 69-72.

[31] Samaniego, F. J (2007). System Signatures and Their Applications in Engineering Reliability (Internat. Ser. Operat. Res. Manag. Sci. 110). Springer, New York.

[32] Samaniego, F. J., Balakrishnan, N. And Navarro, J. (2009). Dynamic signatures and their use in comparing the reliability of new and used systems. Naval Res. Logistics 56, 577-591.

[33] Shaked, M. and Shanthikumar, J. G. (2007). Stochastic Orders. Springer, New York.

[34] Tavangar, M. AND Asadi, M. (2010). A study on the mean past lifetime of the components of $(n-k+1)$-out-of- $n$ system at the system level. Metrika 72, 59-73.

[35] Zhang, Z. (2010). Ordering conditional general coherent systems with exchangeable components. J. Statist. Planning Infer. 140, 454-460. 